\title{
Femtosecond Visualization of hcp-Iron Strength and Plasticity under Shock Compression
}

\author{
Sébastien Merkel@, ${ }^{1, *}$ Sovanndara Hok $\odot,{ }^{2}$ Cynthia Bolme, ${ }^{3}$ Dylan Rittman, ${ }^{2}$ Kyle James Ramos, ${ }^{3}$ Benjamin Morrow, ${ }^{3}$ \\ Hae Ja Lee, ${ }^{4}$ Bob Nagler, ${ }^{4}$ Eric Galtier, ${ }^{4}$ Eduardo Granados $\odot,{ }^{4, \uparrow}$ Akel Hashim, ${ }^{4, \downarrow}$ Wendy L Mao, ${ }^{2}$ and Arianna E Gleason ${ }^{2,4}$ \\ ${ }^{1}$ Univ. Lille, CNRS, INRAE, Centrale Lille, UMR 8207-UMET-Unité Matériaux et Transformations, \\ F-59000 Lille, France \\ ${ }^{2}$ Stanford University, Stanford, California 94305, USA \\ ${ }^{3}$ Los Alamos National Laboratory, Los Alamos, New Mexico 87545, USA \\ ${ }^{4}$ SLAC National Accelerator Laboratory, Menlo Park, California 94025, USA
}

(Received 13 May 2021; revised 28 July 2021; accepted 28 September 2021; published 9 November 2021)

\begin{abstract}
Iron is a key constituent of planets and an important technological material. Here, we combine in situ ultrafast X-ray diffraction with laser-induced shock compression experiments on Fe up to 187(10) GPa and $4070(285) \mathrm{K}$ at $10^{8} \mathrm{~s}^{-1}$ in strain rate to study the plasticity of hexagonal-close-packed (hcp)-Fe under extreme loading states. $\{10 \overline{1} 2\}$ deformation twinning controls the polycrystalline Fe microstructures and occurs within $1 \mathrm{~ns}$, highlighting the fundamental role of twinning in hep polycrystals deformation at high strain rates. The measured deviatoric stress initially increases to a significant elastic overshoot before the onset of flow, attributed to a slower defect nucleation and mobility. The initial yield strength of materials deformed at high strain rates is thus several times larger than their longer-term flow strength. These observations illustrate how time-resolved ultrafast studies can reveal distinctive plastic behavior in materials under extreme environments.
\end{abstract}

DOI: 10.1103/PhysRevLett.127.205501

Understanding the mechanical properties of solid materials under extreme loading conditions is crucial for the formation of planetary bodies [1], ballistics, and penetration [2], or the strengthening of ceramics [3]. Despite considerable computational and experimental effort, however, plasticity models in the high strain rate deformation regime only exist for a few model metals, such as $\mathrm{Ta}$, and require input from experiments [4,5]. Here, we build upon a novel experimental layout and use in situ $\mathrm{x}$-ray diffraction to measure polycrystalline texture and deviatoric stress in situ up to $P-T$ conditions of $199 \mathrm{GPa}$ and $4383 \mathrm{~K}$ at a strain rate on the order of $10^{8} \mathrm{~s}^{-1}$. Our experiment allows measurements of stress and texture at the nanosecond timescale, as a shock progresses into the sample, and identification of the time dependence of plastic flow. This information is critical to feed constitutive models of materials under extreme strain rates, which, until now, have only been available using sequences of multiscale calculations $[4,6]$. We bridge this knowledge gap with measurements of strength and identification of dominant deformation mechanisms for a model engineering structural component and important

Published by the American Physical Society under the terms of the Creative Commons Attribution 4.0 International license. Further distribution of this work must maintain attribution to the author(s) and the published article's title, journal citation, and DOI.
Earth and planetary material: iron (Fe). Pure Fe undergoes several structural transitions at high-pressure and hightemperature $(P-T)$ and serves as an archetype for the most advanced studies regarding the physical properties of materials under extreme conditions [7-10]. At ca. $13 \mathrm{GPa}$, Fe transforms from the body-centered-cubic (bcc) to hcp structure. This transition is martensitic, following the Burgers orientation relationships under hydrostatic conditions [11] and relatively close orientation relationships under shock [12], occurs at subnanosecond timescales $[13,14]$, and is accompanied by stress relaxation with a strong coupling between plasticity and the phase transformation [15-20]. Upon compression and deformation, stress and microstructures develop in the hcp phase of Fe, with important implications regarding anisotropy in the Earth's inner core and technological applications [21-23]. In addition, the hcp phase of Fe and its physical properties are representative of hcp metals with advanced applications, such as $\mathrm{Ti}, \mathrm{Zr}, \mathrm{Mg}, \mathrm{Zn}$, or Be [24], and for which the foundation of multiscale calculations are just being laid out [25-27]. Here, we identify that $\{10 \overline{1} 2\}$ deformation twinning controls the microstructures of hcp-Fe under such conditions. In addition, we observe a fast increase of elastic stresses prior to plastic yielding, actually observed in two experiments. This initial elastic overshoot is a distinctive feature of plastic deformation at high strain rates for which a peak elastic precursor is reached before generating a sufficient defect density for the onset of plastic flow. 
The experiments were performed at the matter at extreme conditions (MEC) end station of the Linac Coherent Light Source (LCLS), SLAC National Accelerator Laboratory. Fe was dynamically compressed to a peak $P-T$ state on the principal Hugoniot via the laser-driven ablation of a Kapton film launching a shock wave into a Fe foil (Fig. 1). Uniform conditions within the sample are achieved within $\sim 1-10 \mathrm{~ns}$ and the sample state is probed using a quasimonochromatic $(d E / E=0.3 \%)$ self-amplified spontaneous emission x-ray beam with energies varying between 6.883 and $6.960 \mathrm{keV}$, an $\mathrm{x}$-ray spot size of $17 \mu \mathrm{m}$ diameter, and diffraction recorded using a single $\mathrm{x}$-ray pulse of $55 \mathrm{fs}$ in duration. Data from the six independent detectors [Fig. 1(a)] are backprojected onto a virtual detector, $60 \mathrm{~mm}$ behind the sample, and reconstructed using material analysis using diffraction (MAUD) [28] assuming a sample consisting of three phases: ambient- $P$ bcc-Fe, compressed bcc-Fe, and compressed hcp-Fe, from which we extract the unitcell parameters, average differential stress, and texture [Fig. 1(c)]. The time dependence of the sample state is determined by repeating the experiment on a different target of the same starting material and collecting data at different times. Additional details on the experiments, data reduction, hydrodynamic simulations, and tabulated results are provided in the Supplemental Material [29].

The experiments are organized into four time series, defined by the $P-T$ conditions and specific optical drive laser parameters. In experiments performed at 187(10) GPa and 4070(285) K (time series 1, Fig. 2), hcp-Fe forms $5.0 \mathrm{~ns}$ after the optical laser drive reaches the ablator. After synthesis, hcp-Fe displays a transformation texture with basal planes parallel to compression. The $c$ axis lies perpendicular to the shock propagation direction, in agreement with static observations of the bcc-to-hep transformation in $\mathrm{Fe}$ [20]. At $0.59 \mathrm{~ns}$ after synthesis, the differential stress in hcp-Fe is 1.4(2.1) GPa. Upon further compression, texture evolves with the appearance of a secondary maximum $30^{\circ}$ off [0001]. This is associated with a stress increase up to 6.5(1.0) GPa. Both orientation distribution maxima at $\langle 2 \overline{110}\rangle$ and $30^{\circ}$ off [0001] in the inverse pole figures remain in the rest of the time series while the stress decreases to 2.0(10.0) GPa 2.52 ns after the synthesis of hcp-Fe, before the start of the pressure release. At conditions of 36(10) GPa and 630(115)K (time series 5, Fig. 2), hcp-Fe is observed after $11.15 \mathrm{~ns}$, with a differential stress of $0.8(1.3) \mathrm{GPa}$ and the same transformation texture. Texture does not evolve significantly upon further compression while the differential stress increases to 10.4(5.1) GPa. The change of texture and drop in stress observed in time series 1 is not observed. Time series 6 at 55(9) $\mathrm{GPa}$ and 875(140) $\mathrm{K}$ shows intermediary results between time series 1 and 5, with the differential stress in hcp-Fe increasing up to 9.9(2.6). Hcp-Fe shows a dominant $\langle 2110\rangle$ transformation texture and the development of a weak secondary maximum $30^{\circ}$ off [0001]. Finally, for time
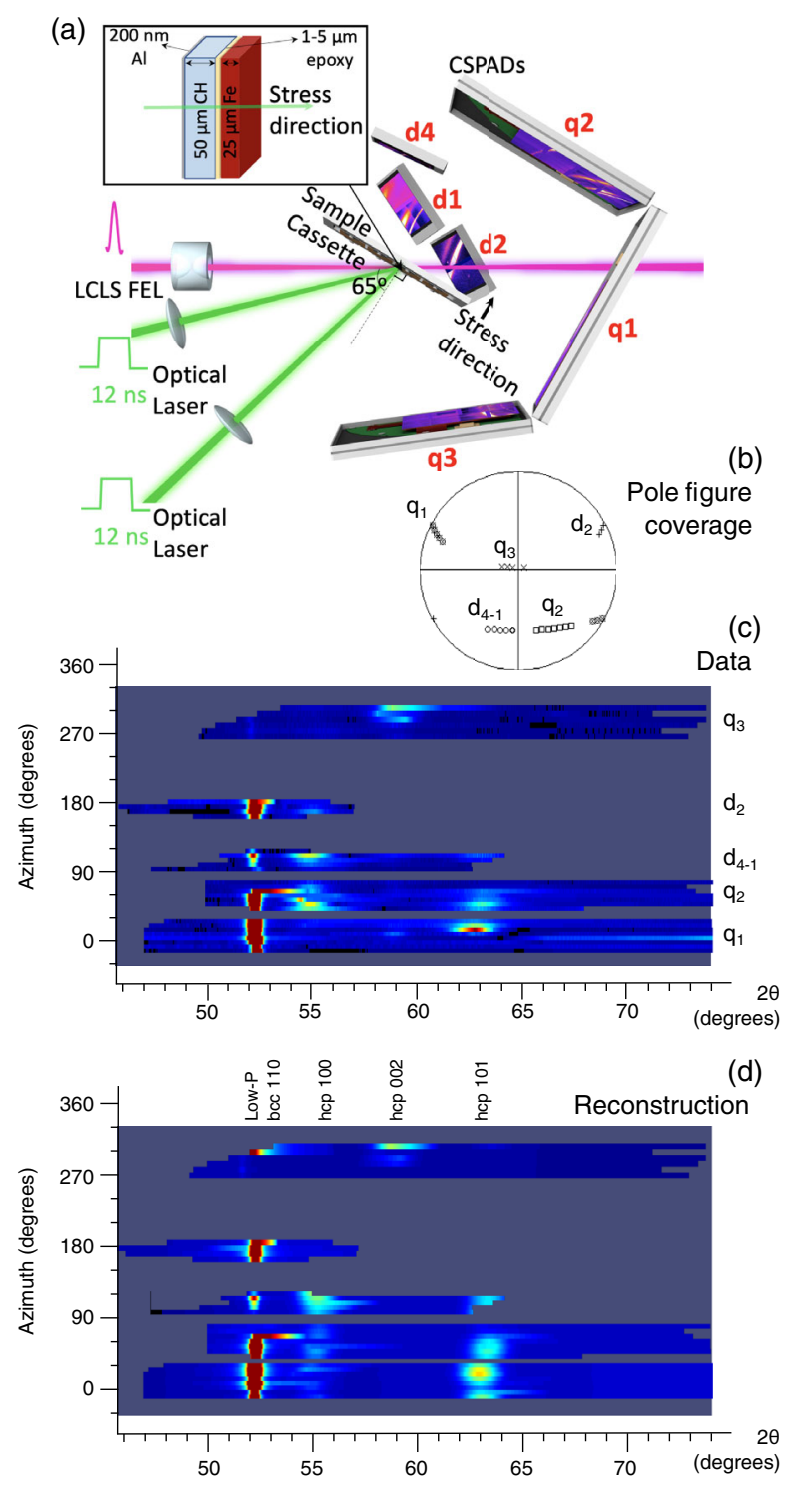

FIG. 1. Geometry for x-ray stress and texture analysis under dynamic compression. (a) The $\mathrm{x}$-ray free-electron laser (XFEL) probe is $65^{\circ}$ away from the shock propagation direction and diffraction measured on six Cornell-SLAC pixel array detectors (CSPADs, $q_{1, \ldots, 3}$ and $d_{1, \ldots, 3}$ ) optimized to cover wider and key directions. Inset: target stack. (b) Pole figure coverage with the compression direction in the center. (c) Original diffraction data at run 149 (193 GPa, $4230 \mathrm{~K}), 1.12 \mathrm{~ns}$ after hcp-Fe synthesis and (d) MAUD reconstruction with labels for the diffraction peaks of the remaining untransformed bcc-Fe and high-pressure hcp-Fe. Variations of peak intensities and positions with orientation are representative of texture and stress, respectively.

series 4, at 23(2) GPa and 494(14) K, we also observe a transformation texture with $c$ axes perpendicular to the shock propagation direction, albeit with a maximum at $\langle 10 \overline{1} 0\rangle$ rather than $\langle 2 \overline{11} 0\rangle$ with the development of a secondary maximum at [0001] upon further compression. Stress is 5.5(3.3) GPa after transformation and decreases later to $1.3(1.4) \mathrm{GPa}$. 


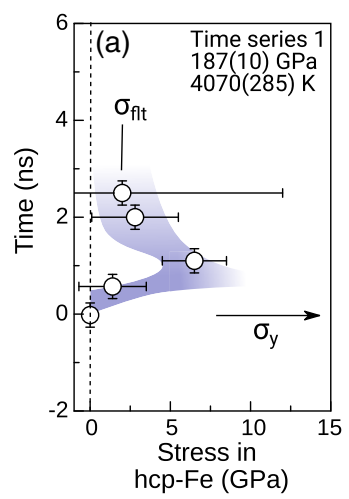

(b)
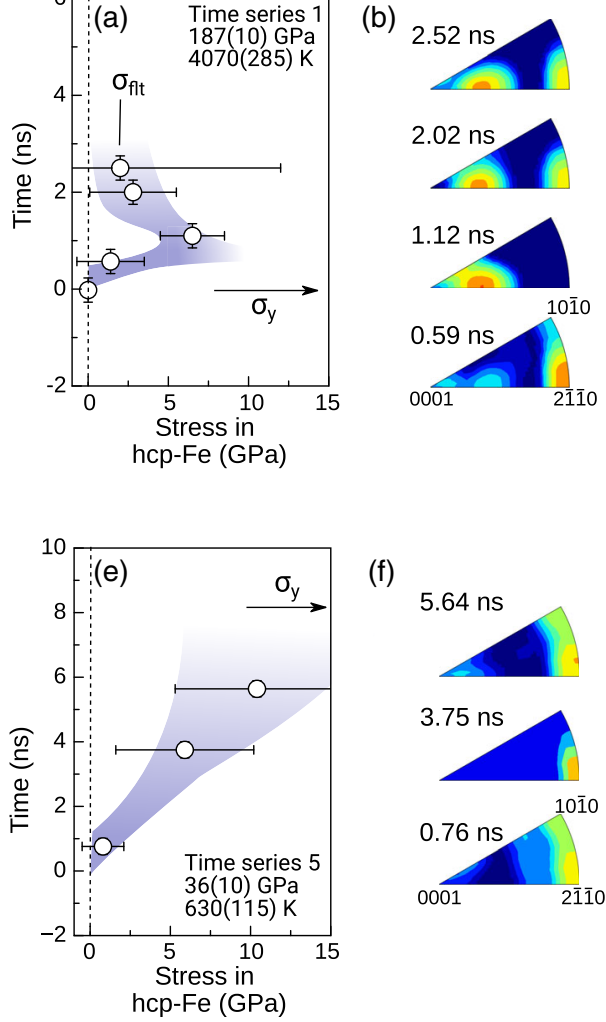
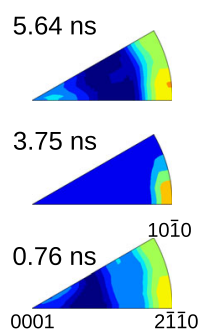
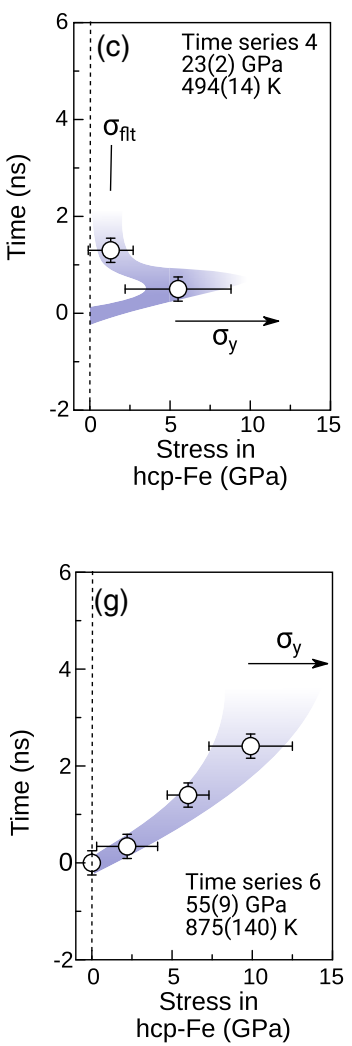

hcp-Fe (GPa) (d)

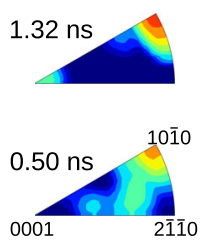

31.6
10.0 3.2

1.0

0.3

(i)

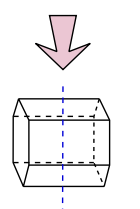

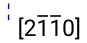
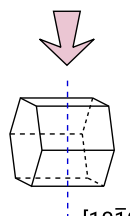

(h)
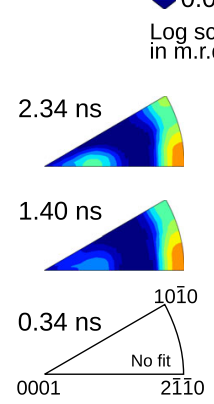

FIG. 2. Results for time series (a),(b) 1, (c),(d) 4, (e),(f) 5, and (g),(h) 6. Times have been rescaled to set $t=0$ when hcp-Fe is formed. (a),(c),(e),(g) Differential stress vs time in hcp-Fe. Circles, experimental data with $\pm 1 \sigma$ error bars; blue shaded areas, guides to the eye through the experimental data. $\sigma_{y}$ indicate potential values for the yield strength and $\sigma_{\mathrm{flt}}$ is the long-term flow strength. (b),(d),(f),(h) Inverse pole figures of the compression direction representing texture in hcp-Fe. We observe components at $\langle 2 \overline{110}\rangle,\langle 10 \overline{1} 0\rangle$, [0001], and approximately $30^{\circ}$ off [0001]. Texture $0.34 \mathrm{~ns}$ after hcp-Fe synthesis in time series 6 could not be fit due to a significant peak overlap with the remaining ambient conditions bcc-Fe. (i) Sketch of hcp-crystals with the shock direction, shown by the pink arrow, aligned with

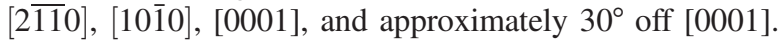

Time series 1 and 4 show a clear hcp-Fe texture evolution. Texture evolution in hcp-Fe was studied at low strain rates $\left(10^{-5}-10^{-3} \mathrm{~s}^{-1}\right)$ and up to $220 \mathrm{GPa}$ at $300 \mathrm{~K}$ [21] and at high $P-T$ of $13-17 \mathrm{GPa}$ and up to $700 \mathrm{~K}$ $[47,48]$. Earlier publications focused on a dominant basal slip [21], while later and more appropriate analysis highlighted the fundamental role of $\{10 \overline{1} 2\}$ tensile twins and pyramidal $\langle c+a\rangle$ slip in experiments [47]. Numerical models of Earth's inner core properties require dominant slip on the pyramidal $\langle c+a\rangle$ slip system to reproduce seismic observations [49], probably due to high temperatures and slow deformation conditions. Here, we use the Los Alamos viscoplastic self-consistent (VPSC) model [50] assuming a 3000 spherical grains aggregate and the affine interaction (Fig. 3). The results are unambiguous: the secondary maximum $30^{\circ}$ off [0001] [Fig. 2(b)] is formed by the activation of $\{10 \overline{1} 2\}$ deformation twins in grains initially oriented with $\langle 2 \overline{11} 0\rangle$ parallel to compression [Fig. 3(a)]. The simulation predicts a decrease of intensity at $\langle 2 \overline{11} 0\rangle$ with deformation, in contrast with experiments. This arises from new hcp-Fe grains being formed with a dominant transformation texture as the shock front advances through the material. VPSC simulations with dominant $\{10 \overline{1} 2\}$ twinning are equally successful at modeling results in time series 4 , accounting for the slightly different starting texture [Figs. 2(d) and 3(b)].

Texture does not evolve in time series 5 and only marginally in time series 6. Texture evolution requires (i) sufficient stress to overcome the elastic limit or yield strength of the material and (ii) sufficient plastic strain to induce noticeable microstructural changes. In both time series 5 and 6 , we observe a continuous increase of differential stress with time and little or no texture evolution. In time series 1 and 4, the measured stress increases and decreases, in association with texture evolution. Thus, stress in time series 1 and 4 did overcome the yield strength barrier of hcp-Fe, while it did not in time series 5 and 6 .

Texture evolution is indicative of active plastic deformation, reflective of ongoing stress relief in the polycrystal and related to a fundamental property of materials: strength. Understanding and modeling strength and its dependence on pressure, temperature, and strain rate typically involves a sequence of multiscale calculations, from first principles to dislocation dynamics to continuum modeling, that has 

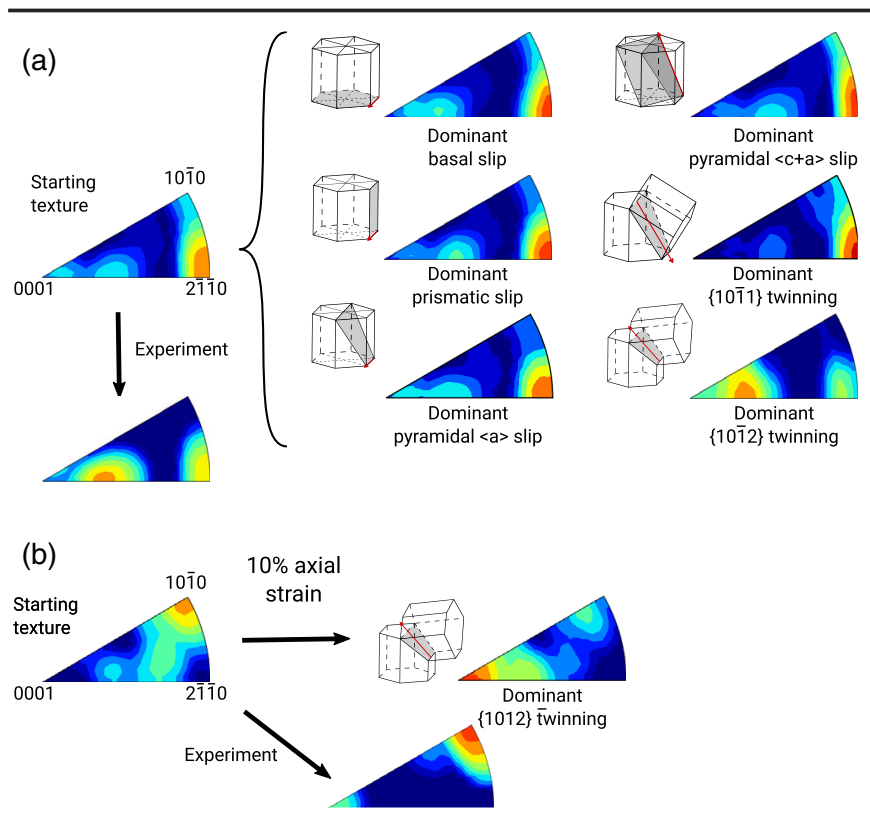

FIG. 3. Viscoplastic self-consistent simulations for texture evolution in hcp-Fe. (a) Inverse pole figure of the compression direction after $10 \%$ axial plastic strain and easy basal, prismatic, pyramidal $\langle a\rangle$, and pyramidal $\langle c+a\rangle$ slip, in addition to $\{10 \overline{1} 1\}$ compression and $\{10 \overline{1} 2\}$ extension twinning starting from the transformation texture in time series 1. (b) Simulation with dominant $\{10 \overline{1} 2\}$ twinning and starting from the transformation texture in time series 4 .

only been undertaken for simple cubic metals such as Ta or Va [4] or $\mathrm{Cu}$ [6] or oxides such as $\mathrm{MgO}$ [51] and lacks experimental validation at the most extreme conditions. Based on model results on simple metals [4,6,52], for strain rates below $\sim 10^{7} \mathrm{~s}^{-1}$, stress increases linearly with time (or strain) until the transition from elastic to plastic deformation. The material then hardens due to an increased defect density until a long-term flow strength or steady-state strength is reached, typically after $10 \%$ axial strain. Above $\sim 10^{7} \mathrm{~s}^{-1}$, there is an elastic overshoot in the initial stress response due to an insufficient quantity of defects to accommodate plastic strain and kinetics for defect motion in the phonon-drag-dominated regime $[4,6]$. The material deforms elastically until sufficient defects have been generated, which then decrease the stress. Under such conditions, the material reaches a maximum yield strength at the elastic-plastic transition. Later, stress decreases and saturates at the long-term flow strength. Based on Fig. 2, our experiments overcame the yield strength barrier of hcpFe in time series 1 and 4, within less than $1 \mathrm{~ns}$, and not in time series 5 and 6 . The optical drive profile to generate pressure was much sharper in the early stages of compression in time series 1 than in all other time series (Supplemental Material, Fig. S2 [29]). Stress increase was hence much faster for time series 1 and sufficient to overcome the yield strength barrier, while it was slower in time series 5 and 6 (Fig. 2). Time series 4 was performed at a lower pressure, with a lower yield strength, that was hence overcome despite the slower drive profile. The exact yield strength value cannot be determined, due to a lack of time-resolved measurements and integrated $\mathrm{x}$-ray diffraction over hcp-Fe grains in a different state, but is estimated to be above all values measured here, 6.5(1.0) GPa for $P-T$ conditions of 187(10) GPa and 4070(285) K. The longterm flow strength of Fe at 187(10) GPa and 4070(285) K, however, is much lower, on the order of 3(2) GPa. As for time series 5, we can deduce that the experiment did not provide sufficient strain to overcome the yield strength barrier, which is above 10.4(5.1) GPa at 36(10) GPa and 630(115) K. For time series 6 at 55(9) GPa and 875(140) K, we do not observe a decrease of stress following the initial elastic overshoot and stresses reach 9.9(2.6) GPa. In time series 4 at 23(2) $\mathrm{GPa}$ and 494(14) $\mathrm{K}$, the maximum measured stress is 5.5(3.3) GPa.

Static strength measurements (i.e., the long-term flow strength at static experiments strain rates) are between 7 and $21 \mathrm{GPa}$ at $300 \mathrm{GPa}$ and $300 \mathrm{~K}[23,53]$. Based on temperature measurements during laser-driven compression and a simple plastic heating model, the strength of hcpFe was estimated to be $\approx 100 \mathrm{GPa}$ at $550 \mathrm{GPa}$ at shock strain rates [22]. Our measurements indicate that the longterm flow strength of $\mathrm{Fe}$ at $187(10) \mathrm{GPa}$ and 4070(285) $\mathrm{K}$ is on the order of 3(2) GPa, 2 orders of magnitude below this estimate, and closer to static measurements (Fig. 4). We explain this discrepancy by noticing two main differences: (i) Reference [22] assigned temperature increase to the initial shock and plastic work with a simple steady-state flow law that does not account for time-dependent strength (e.g., Fig. 2) and other sources of heat [54]. (ii) X-ray diffraction averages the stress state in the whole probed sample, which may be in a different state between the shock front and the bulk of the high-pressure phase. Our maximum stress measurement at 187(10) GPa and 4070(285) K is 6.5(1.0) GPa. According to models for $\mathrm{Cu}, \mathrm{Ta}$, or $\mathrm{Va}$ $[4,6]$, the maximum yield strength at $\sim 10^{7}-10^{9} \mathrm{~s}^{-1}$ may be 2-5 times larger than the long-term flow strength. As such, the yield strength at the initial onset of plastic flow may be larger than measured here, between our estimate and that of Ref. [22].

There is significant ongoing interest in the development of constitutive models for the deformation of solids as a function of $P, T$, and strain rate. Predictions of plastic flow under extreme states of loading are currently only achievable for model materials. They also require input and validation against experimental data regarding strength, plastic mechanisms, and their dependence on time and strain rate, which we provide here for hcp-Fe. Our results highlight the relevance of twinning for hcp metal plasticity. Twinning initiation requires the nucleation and growth of twin embryo and we show that it can occur within $1 \mathrm{~ns}$, with measurable effects on microstructures at the polycrystal scale. Stress later decreases through plastic 


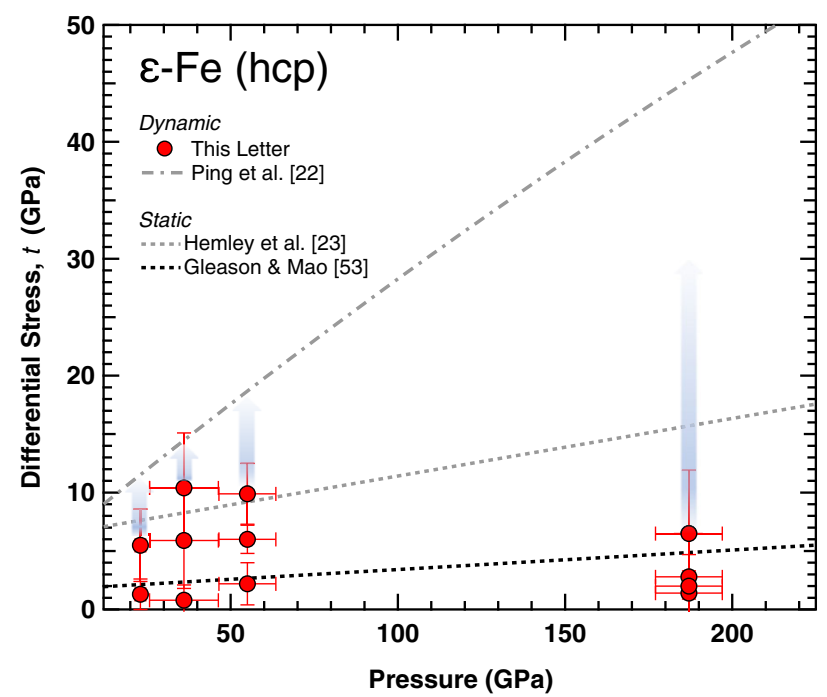

FIG. 4. Static and dynamic strength of hcp-Fe at high $P-T$. Trend lines, Refs. [22,23,53]; red circles, this Letter. The timedependent stresses recorded here in situ using X-ray diffraction plot far below previous dynamic compression estimates and closer to static measurements at $300 \mathrm{~K}$. For strain rates above $10^{7} \mathrm{~s}^{-1}$, however, the true yield strength of $\mathrm{Fe}$ - the stress at the elastic-to-plastic transition-may be several times larger than the longer-term flow strength, which is highlighted by the blue arrows. This is due to an insufficient quantity of defects to accommodate plastic strain in the initial compression and a phonon-drag-controlled defect kinetics.

relaxation, again within a timescale of $\approx 1 \mathrm{~ns}$, consistent with observations on Ta [55].

Our results also demonstrate the importance of timescales in the definition of material strength, from the initial yield strength at the onset of the elastic-to-plastic transition, to the long-term flow strength once plastic flow is fully achieved. Stress measurement could be improved with the addition of time-dependent measurements in Fig. 2, which is already achievable with current experimental layouts. The characterization of the true yield strength would also require spatially resolved $\mathrm{x}$-ray diffraction measurements to differentiate between stress at the shock front and that in the bulk material. This, however, would require significant development. Finally, one would need to differentiate between the stress at elastic-to-plastic transition and the stress required for twin nucleation, which we were not able to address here. Nevertheless, future time-resolved studies of plastic flow will open new doors toward building predictive capabilities for modeling material strength and plasticity in extreme environments, with relevant implications that range from modeling the dynamics and formation of planets to the design of novel materials for advanced applications.

The raw diffraction images for this Letter are openly available from the Zenodo data archive [56].

S. M. performed part of this work as a Blaustein Visiting Professor at Stanford University. He received support from the MetalCore grant, which was supported by the French government through the Programme Investissement d'Avenir (I-SITE ULNE R-ERCGEN-19-006-MERKEL / ANR-16-IDEX-0004 ULNE) managed by the Agence Nationale de la Recherche. A. E. G. acknowledges funding support from Los Alamos National Laboratory (LANL) Reines Laboratory Directed Research and Development (LDRD) and U.S. Department of Energy Early Career Award in Fusion Energy Sciences. A. E. G. and W. M. both acknowledge support from the NSF Geophysics Program. C. B., K. J. R., and B.M. acknowledge funding support from LANL Science Campaign 2, Dynamic Materials Properties Program. The Matter in Extreme Conditions (MEC) instrument at the Linac Coherent Light Source (LCLS) at the SLAC National Accelerator Laboratory, B. N., E. G., A. H, and H. J.L are supported by the U.S. Department of Energy, Office of Science, Office of Fusion Energy Sciences under Award No. DE-AC02-76SF00515.

*sebastien.merkel@univ-lille.fr

Present address: CERN, 1211 Genève 23, Switzerland.

"Present address: Quantum Nanoelectronics Laboratory, Department of Physics, University of California at Berkeley, Berkeley, California 94720, USA.

[1] J. Monteux and J. Arkani-Hamed, Icarus 331, 238 (2019).

[2] S. Muskeri, D. Choudhuri, P. A. Jannotti, B. E. Schuster, J. T. Lloyd, R. S. Mishra, and S. Mukherjee, Adv. Eng. Mater. 22, 2000124 (2020).

[3] P. Shukla, R. Crookes, and H. Wu, Mater. Des. 167, 107626 (2019).

[4] N. R. Barton, J. V. Bernier, R. Becker, A. Arsenlis, R. Cavallo, J. Marian, M. Rhee, H.-S. Park, B. A. Remington, and R. T. Olson, J. Appl. Phys. 109, 073501 (2011).

[5] C. E. Wehrenberg, D. McGonegle, C. Bolme, A. Higginbotham, A. Lazicki, H. J. Lee, B. Nagler, H.-S. Park, B. A. Remington, R. E. Rudd, M. Sliwa, M. Suggit, D. Swift, F. Tavella, L. Zepeda-Ruiz, and J. S. Wark, Nature (London) 550, 496 (2017).

[6] B. L. Hansen, I. J. Beyerlein, C. A. Bronkhorst, E. K. Cerreta, and D. Dennis-Koller, Int. J. Plast. 44, 129 (2013).

[7] S. Anzellini, A. Dewaele, M. Mezouar, P. Loubeyre, and G. Morard, Science 340, 464 (2013).

[8] Z. Konôpková, R. S. McWilliams, N. Gómez-Pérez, and A. F. Goncharov, Nature (London) 534, 99 (2016).

[9] R. F. Smith, D. E. Fratanduono, D. G. Braun, T. S. Duffy, J. K. Wicks, P. M. Celliers, S. J. Ali, A. Fernandez-Pañella, R. G. Kraus, D. C. Swift, G. W. Collins, and J. H. Eggert, Nat. Astron. 2, 452 (2018).

[10] S. Ritterbex and T. Tsuchiya, Sci. Rep. 10, 6311 (2020).

[11] A. Dewaele, C. Denoual, S. Anzellini, F. Occelli, M. Mezouar, P. Cordier, S. Merkel, M. Véron, and E. Rausch, Phys. Rev. B 91, 174105 (2015).

[12] J. A. Hawreliak and S. J. Turneaure, J. Appl. Phys. 129, 135901 (2021)

[13] J. C. Crowhurst, B. W. Reed, M. R. Armstrong, H. B. Radousky, J. A. Carter, D. C. Swift, J. M. Zaug, R. W. 
Minich, N. E. Teslich, and M. Kumar, J. Appl. Phys. 115, 113506 (2014).

[14] H. Hwang et al., Sci. Adv. 6, eaaz5132 (2020).

[15] D. H. Kalantar et al., Phys. Rev. Lett. 95, 075502 (2005).

[16] K. Wang, S. Xiao, H. Deng, W. Zhu, and W. Hu, Int. J. Plast. 59, 180 (2014).

[17] B. J. Jensen, G. T. Gray, and R. S. Hixson, J. Appl. Phys. 105, 103502 (2009).

[18] A. Vattré and C. Denoual, J. Mech. Phys. Solids 131, 387 (2019).

[19] H.-T. Luu, R. J. Ravelo, M. Rudolph, E. M. Bringa, T. C. Germann, D. Rafaja, and N. Gunkelmann, Phys. Rev. B 102, 020102(R) (2020).

[20] S. Merkel, A. Lincot, and S. Petitgirard, Phys. Rev. B 102, 104103 (2020).

[21] H.-R. Wenk, S. Matthies, R. J. Hemley, H. K. Mao, and J. Shu, Nature (London) 405, 1044 (2000).

[22] Y. Ping, F. Coppari, D. G. Hicks, B. Yaakobi, D. E. Fratanduono, S. Hamel, J. H. Eggert, J. R. Rygg, R. F. Smith, D. C. Swift, D. G. Braun, T. R. Boehly, and G. W. Collins, Phys. Rev. Lett. 111, 065501 (2013).

[23] R. J. Hemley, H. K. Mao, G. Shen, J. Badro, P. Gillet, M. Hanfland, and D. Häusermann, Science 276, 1242 (1997).

[24] T. B. Britton, F. P. E. Dunne, and A. J. Wilkinson, Proc. R. Soc. A 471, 20140881 (2016).

[25] H. Fan, S. Aubry, A. Arsenlis, and J. A. El-Awady, Acta Mater. 92, 126 (2015).

[26] S. Aubry, M. Rhee, G. Hommes, V. Bulatov, and A. Arsenlis, J. Mech. Phys. Solids 94, 105 (2016).

[27] M. C. Messner, M. Rhee, A. Arsenlis, and N. R. Barton, Model. Simul. Mater. Sci. Eng. 25, 044001 (2017).

[28] L. Lutterotti, R. Vasin, and H.-R. Wenk, Powder Diffr. 29, 76 (2014).

[29] See Supplemental Material at http://link.aps.org/ supplemental/10.1103/PhysRevLett.127.205501 for additional details on the experiments, data reduction, hydrodynamic simulations, and tabulated results, which also includes Refs. [30-46].

[30] J. M. Brown and R. G. McQueen, J. Geophys. Res. 91, 7485 (1986).

[31] A. K. Singh, C. Balasingh, H. K. Mao, R. J. Hemley, and J. Shu, J. Appl. Phys. 83, 7567 (1998).

[32] X. Sha and R. E. Cohen, Phys. Rev. B 81, 094105 (2010).

[33] P. A. Turner and C. N. Tomé, Acta Metal. Mater. 42, 4143 (1994).

[34] B. Clausen, C. N. Tomé, D. W. Brown, and S. R. Agnew, Acta Mater. 56, 2456 (2008).

[35] C. J. Neil, J. A. Wollmershauser, B. Clausen, C. N. Tomé, and S. R. Agnew, Int. J. Plast. 26, 1772 (2010).
[36] H. Wang, P. D. Wu, C. N. Tomé, and Y. Huang, J. Mech. Phys. Solids 58, 594 (2010).

[37] S. Merkel, C. N. Tomé, and H.-R. Wenk, Phys. Rev. B 79, 064110 (2009).

[38] P. Raterron, S. Merkel, and C. W. Holyoke III, Rev. Sci. Instrum. 84, 043906 (2013).

[39] S. B. Brown, A. Hashim, A. Gleason, E. Galtier, I. Nam, Z. Xing, A. Fry, A. MacKinnon, B. Nagler, E. Granados, and H. J. Lee, Rev. Sci. Instrum. 88, 105113 (2017).

[40] R. A. Graham and W. P. Brooks, J. Phys. Chem. Solids 32, 2311 (1971).

[41] K.-I. Kondo and T. J. Ahrens, Geophys. Res. Lett. 10, 281 (1983).

[42] J. Wackerle, J. Appl. Phys. 33, 922 (1962).

[43] A. C. Mitchell and W. J. Nellis, J. Appl. Phys. 52, 3363 (1981).

[44] R. G. McQueen, S. P. Marsh, J. W. Taylor, J. N. Fritz, and W. J. Carter, in High Velocity Impact Phenomena, edited by R. Kinslow (Academic Press, San Diego, CA, 1970), pp. 296-417.

[45] J. J. MacFarlane, I. E. Golovkin, and P. R. Woodruff, J. Quant. Spectrosc. Radiat. Transfer 99, 381 (2006).

[46] R. M. More, K. H. Warren, D. A. Young, and G. B. Zimmerman, Phys. Fluids 31, 3059 (1988).

[47] S. Merkel, M. Gruson, Y. Wang, N. Nishiyama, and C. N. Tomé, Model. Simul. Mater. Sci. Eng. 20, 024005 (2012).

[48] Y. Nishihara, T. Ohuchi, T. Kawazoe, Y. Seto, G. Maruyama, Y. Higo, K.-I. Funakoshi, Y. Tange, and T. Irifune, Earth Planet. Sci. Lett. 490, 151 (2018).

[49] A. Lincot, P. Cardin, R. Deguen, and S. Merkel, Geophys. Res. Lett. 43, 1084 (2016).

[50] R. A. Lebensohn and C. N. Tomé, Acta Metal. Mater. 41, 2611 (1993).

[51] P. Cordier, J. Amodeo, and P. Carrez, Nature (London) 481, 177 (2012).

[52] R. F. Smith, J. H. Eggert, R. E. Rudd, D. C. Swift, C. A. Bolme, and G. W. Collins, J. Appl. Phys. 110, 123515 (2011).

[53] A. E. Gleason and W. L. Mao, Nat. Geosci. 6, 571 (2013).

[54] D. J. Luscher, M. A. Buechler, D. J. Walters, C. Bolme, and K. J. Ramos, Int. J. Plast. 111, 188 (2018).

[55] C. E. Wehrenberg, A. J. Comley, N. R. Barton, F. Coppari, D. Fratanduono, C. M. Huntington, B. R. Maddox, H.-S. Park, C. Plechaty, S. T. Prisbrey, B. A. Remington, and R. E. Rudd, Phys. Rev. B 92, 104305 (2015).

[56] S. Merkel, S. Hok, C. Bolme, D. Rittman, K. J. Ramos, H. J. Lee, B. Nagler, E. Galtier, E. Granados, A. Hashim, W. L. Mao, and A. E. Gleason, Zenodo, https://doi.org/10.5281/ zenodo.5526703 (2021). 